Conclusions These data indicate there is no significant difference between ILD and COPD patients' walking distances. ILD patients with a reduced exercise tolerance should be included and referred to PR programmes.

\section{P42 DOES PULMONARY REHABILITATION IMPROVE EXERCISE CAPACITY AND HRQOL IN PATIENTS WITH ASTHMA?}

RJ Tanner, AK Boutou, Y Raste, VM Lord, L Grillo, A Menzies-gow, J Hull, NS Hopkinson ; Royal Brompton Hospital, London, UK

\subsection{6/thoraxjnl-2013-204457.192}

Introduction Pulmonary rehabilitation (PR) benefits patients with COPD, however to date there is limited evidence demonstrating its effectiveness in patients with chronic asthma.

We hypothesised that patients with asthma would have a significant improvement in exercise capacity and HRQOL following a standard PR programme. The aim of this study was a) to evaluate the impact of PR on exercise capacity and HRQoL in patients with asthma and b) to identify the factors influencing attendance and completion.

Methods We retrospectively audited PR outcome in a cohort of asthmatic patients referred for PR at the Royal Brompton Hospital between 2008 and 2012. Exercise capacity (ISWT), quadriceps maximal volitional contraction (QMVC) and HRQoL (HADS, SGRQ, LINQ, MRC) were assessed before and following PR. Logistic regression analysis was used to determine factors predicting PR completion.

Results 49 patients were referred of whom 25 completed $(69.2 \%$ female; mean age: $58.7 \pm 12.5$ years; mean BMI: $31.27 \pm 30.1$ ) Completers were moderately obstructed (mean $\mathrm{FEV}_{1} \%$ pred: $\left.57 \pm 22.9, \mathrm{FEV}_{1} / \mathrm{FVC}: 0.63 \pm 0.14\right) .4 \%$ of them were current smokers and $8 \%$ were ex-smokers (mean history of 11.25 pack/yrs). Mean classes attended were $15.31 \pm 5.4$ per patient. There was a significant improvement in QMVC $(\mathrm{p}=0.02)$ and ISWT $(\mathrm{p}=0.036)$ following PR. Other than LINQ score $(\mathrm{p}=0.016)$, there was no improvement in HRQoL measures following PR. Patients with a higher\%Fat, according to bioelectrical impedance, were less likely to complete PR once they had been referred. No other statistically significant differences were found between completers and non-completers.

Conclusions Asthmatic patients benefit from attending a PR course in terms of functional capacity and muscle strength. Further prospective studies utilising asthma specific quality of life indices are required to evaluate disease specific benefits of PR and delineate factors predicting successful PR.

Does pulmonary rehabilitation improve exercise capacity and HRQoL in patients withasthma?

\begin{tabular}{lll}
\multicolumn{3}{l}{ Abstract P42 Table 1. Patient characteristics pre and post PR. } \\
\hline & Pre PR & Post PR \\
\hline Number & 25 & 25 \\
ISWT (m) & $220(210)$ & $370(302)^{*}$ \\
Best QMVC (kg) & $21.8(10)$ & $24.4(9.9)^{*}$ \\
HAD A & $8.85(4.3)$ & $8.63(5.1)$ \\
HAD D & $6(5)$ & $6(7)$ \\
SGRQ Total score & $49.6(14.1)$ & $48.9(14.9)$ \\
LINQ & $7.85(2.86)$ & $6.11(2.5)^{*}$ \\
MRC & $3.23(0.92)$ & $3.08(0.68)$ \\
\hline Data presented as Mean (SD) or Median (IQR). ${ }^{*}$ P $<0.05$ &
\end{tabular}

${ }^{1} \mathrm{SL}$ Fleming, ${ }^{2} \mathrm{SE}$ Jones, ${ }^{3} \mathrm{~S}$ Green, ${ }^{4} \mathrm{AL}$ Clark, ${ }^{3} \mathrm{C}$ Howe, ${ }^{2}$ SSC Kon, ${ }^{5} \mathrm{M}$ Dickson, ${ }^{1} \mathrm{~J}$ Godden, ${ }^{3}$ D Bell, ${ }^{5}$ BM Haselden, ${ }^{1}$ WD-C Man; ${ }^{1}$ Royal Brompton \& Harefield NHS Foundation Trust, London, UK; ${ }^{2}$ Respiratory NIHR Biomedical Research Unit, Royal Brompton \& Harefield NHS Foundation Trust and Imperial College, London, UK; ${ }^{3}$ NIHR CLAHRC for Northwest London, London, UK; ${ }^{4}$ Harefield Pulmonary Rehabilitation Unit, Royal Brompton \& Harefield NHS Foundation Trust, Harefield, UK; ${ }^{5}$ The Hillingdon Hospitals NHS Foundation Trust, Uxbridge, UK

\subsection{6/thoraxjnl-2013-204457.193}

Background Early post-hospitalisation pulmonary rehabilitation (PR) following acute exacerbation of COPD (AECOPD) improves health-related quality of life, increases exercise capacity and reduces rate of hospital readmission. However, only a minority of eligible patients are referred to or receive this intervention (Jones et al Thorax 2013). We explored patient acceptability for post-AECOPD PR and the referral process through face-to-face audio- and video-taped interviews.

Methods Ten patients were interviewed using experience based co-design (EBCD) methodology: six PR “completers”, one PR starter who subsequently withdrew, and 3 patients who declined $\mathrm{PR}$. The films were analysed and edited to represent the common themes. A patient-staff event was held to co-design a patient information leaflet and video.

Results Overall, the patient completers were positive about their experience. They most liked: the atmosphere; the equipment; group social interaction; doing more exercise than they thought they could; learning how to manage their lung condition. They least liked: getting there; being 'shattered' afterwards; no tea and coffee break; no introductions. For patients who declined, the reasons given were: "I don't know what rehab is ...... noone has explained it"; "I was never offered rehab"; "It is too far away- I would go if transport was paid for." A recurring theme was that patients had poor recall of information provided during hospital admission.

Conclusion The findings highlighted the complexity of interactions between patient and healthcare professionals. In response to this, an information leaflet and video are being produced using the filmed interviews and feedback from the patient staff co-design event to facilitate the referral process. The best timing Other themes were improving PR accessibility and enhancing social aspects of PR. Two new community sites were opened to provide wider coverage of the borough andrefreshments are now offered routinely during education classes. Furthermore, patients and staff are introduced to each other routinely at the beginning of PR classes. 159 referrals for post-AECOPD PR have been made in the past 18 months and patient satisfaction rates are 98\%. The EBCD approach was ideal in engaging patients in the co-design of service improvements.

\section{P44 MAINTENANCE OF SPACE FOR COPD (A SELF- MANAGEMENT PROGRAMME OF ACTIVITY, COPING \& EDUCATION): A SIX MONTH QUALITATIVE STUDY}

${ }^{1}$ LD Apps, ${ }^{1}$ SL Harrison, ${ }^{1} K E$ Mitchell, ${ }^{1} \mathrm{M}$ Steiner, ${ }^{1} \mathrm{M}$ Morgan, ${ }^{1} \mathrm{SJ}$ Singh, ${ }^{2} \mathrm{~S} J$ Singh; ${ }^{1}$ Centre For Exercise \& Rehabilitation Science, Respiratory Biomedical Research Unit, University Hospitals of Leicester NHS Trust, Leicester, Leicestershire; ${ }^{2}$ Faculty of Health \& Life Sciences, Coventry University, Coventry, Warwickshire

10.1136/thoraxjn-2013-204457.194 and delivery of patient information is currently being evaluated. 